Original Paper

\title{
Mathematical Model for the Effect of Blade Friction on the Performance of Pelton Turbine
}

\author{
Iresha Udayangani Atthanayake ${ }^{1}$, Thusitha Sugathapala ${ }^{2}$ and Rathna Fernando ${ }^{3}$ \\ ${ }^{1}$ Department of Mechanical Engineering, The Open University of \\ Sri Lanka, Nawala, 1025, Sri lanka, ireshairesha@yahoo.com, \\ ${ }^{2}$ Department of Mechanical Engineering, University of Moratuwa \\ Moratuwa, 1025, Sri lanka, thusitha@mech.mrt.ac.lk \\ ${ }^{3}$ Department of Mechanical Engineering, \\ University of Moratuwa, Moratuwa ,1025, Sri Lanka,marv_f@hotmail.com.
}

\begin{abstract}
Water turbines have been used in electricity generation for well over a century. Hydroelectricity now supplies $19 \%$ of world electricity. Many hydro power plants are operated with Pelton turbines, which is an impulse turbine. The main reasons for using impulse turbines are that they are very simple and relatively cheap. As the stream flow varies, water flow to the turbine can be easily controlled by changing the number of nozzles or by using adjustable nozzles. Scientific investigation and design of turbines saw rapid advancement during last century. Most of the research that had been done on turbines were focused on improving the performance with particular reference to turbine components such as shaft seals, speed increasers and bearings. There is not much information available on effects of blade friction on the performance of turbine. The main focus in this paper is to analyze the performance of Pelton turbine particularly with respect to their blade friction.
\end{abstract}

Keywords: Pelton Turbine, Blade friction, Direct friction , Indirect friction

\section{Introduction}

Pelton turbine is an impulse turbine. The runner of the Pelton turbine consists of double hemispherical cups fitted on its periphery. The jet strikes these cups at the central dividing edge of the front edge. The central dividing edge is also called as splitter. The water jet strikes edge of the splitter symmetrically and equally distributed into the two halves of hemispherical bucket The inlet angle of the jet is therefore between $1^{\circ}$ and $3^{\circ}$. Theoretically if the buckets are exactly hemispherical it would deflect the jet through $180^{\circ}$. Then the relative velocity of the jet leaving the bucket would be opposite in direction to the relative velocity of the jet entering. This cannot be achieved practically because the jet leaving the bucket then strikes the back of the succeeding bucket and hence overall efficiency would decrease. Therefore in practice the angular deflection of the jet in the bucket is limited to about $165^{\circ}$ or $170^{\circ}$, and the bucket is slightly smaller than a hemisphere in size. The amount of water discharges from the nozzle is regulated by a needle valve provided inside the nozzle. One or more water jets can be provided with the Pelton turbine depending on the requirement.

There are several key components of the turbine design that determine the power capabilities of the system. This includes the design head, the discharge, the runner throat diameter, and the runner rotational speed. The power capability of the unit is also limited by losses. Due to the mechanics of the system, several types of losses are expected. First, frictional losses occur as water flows across the various surfaces of the system. Secondly, inlet and bend losses occur as the water is forced through the geometry of the intake, scroll case, and draft tubes. And finally, the motion of the mechanical parts of the turbine results in mechanical losses. The sum of these losses plus the energy removed for power equals the head drop through the turbine unit. The basic equations that can be applied to an any turbine are as follows.

The power (P) of any rotary hydraulic machine depends upon the density of flowing fluid $(\rho)$, speed(N), the characteristic diameter(D), the head change $(\Delta \mathrm{H})$, the volume flow rate $(\mathrm{Q})$ and the gravitational constant $(\mathrm{g})$. From the dimensional analysis three non dimensional numbers can be obtained and can be written as 
$\frac{P}{\rho N^{3} D^{5}}=\phi\left(\frac{Q}{N D^{2}}\right)\left(\frac{g H}{N^{2} D^{2}}\right)$

Where $\frac{P}{\rho N^{3} D^{5}}$ - power coefficient

$$
\begin{aligned}
& \left(\frac{Q}{N D^{2}}\right) \text { - flow coefficient } \\
& \left(\frac{g H}{N^{2} D^{2}}\right) \text { - head coefficient }
\end{aligned}
$$

All these equations provide rough estimate of power developed by a turbine. But in the case of exact or approximate power calculations all the losses incurred within the flow system is necessary. Presently performance prediction of hydraulic turbines, such as efficiency and dynamic behavior under different operating conditions, is of high interest to researchers. In many research it has been revealed that the blade friction of the Pelton turbine plays an important role in the performance prediction. But there is no mathematical model to calculate exact figure of power loss or even approximate value.

\section{Basic Velocity triangles}

Inlet

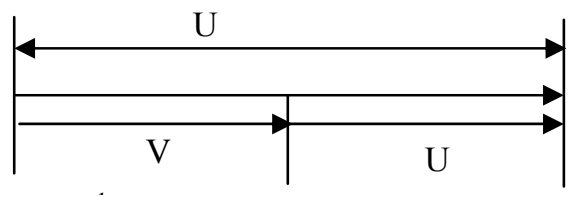

Outlet

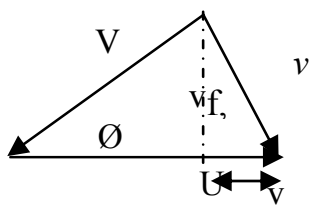

Fig. 1 Basic velocity triangles

Force exerted by water $=\dot{m}\left(v_{w, i}-v_{w, o}\right)$

Work done/Power(P) $=\dot{m}\left(v_{w, i}-v_{w, o}\right) U_{1}$

Hydraulic efficiency $=\frac{\left(v_{w, i}-v_{w, o}\right) \frac{U_{1}}{g}}{\frac{U^{2}}{2 g}}$

Condition for Maximum hydraulic efficiency of Pelton Wheel

$v_{w, i}=U=V_{1}+U_{1}$

$v_{w, o}=U_{1}-v_{r, o} \cos \phi$

$\therefore P=\dot{m}\left(v_{r, i}+v_{r, o} \cos \phi\right)$

If there is no frictional resistance along the vanes

$\left(v_{r, i}=v_{r, o}\right)$

$\therefore P=\dot{m} U_{1}\left(U-U_{1}\right)(1+\cos \phi)$

$\frac{d P}{d U_{1}}=\dot{m}\left(U-2 U_{1}\right)(1+\cos \phi)$

$\mathrm{P}$ to be maximum $\frac{d P}{d U_{1}}=0$

$\therefore U=2 U_{1}$

In practical situations the friction in the nozzle and friction in the buckets of the Pelton wheel plays an important role. Therefore following empirical relationships are used in designing Pelton Wheels for given conditions.

- $\quad$ Velocity of jet $=0.98$ to $0.99 \sqrt{2 g H}$

- Velocity of the runner at pitch diameter $=0.44$ to $0.46 \sqrt{2 g H}$

- Angle through which water is deflected in buckets $=165^{\circ}$

- Axial width of buckets $=3.5$ to $4 \times$ diameter of the jet

- $\quad$ Number of buckets $=\left(\frac{D}{d}+15\right)$

- Ratio of pitch diameter of runner to jet diameter is not less than 12 . 


\section{Literature Available}

Daugherty [3] has analyzed the performance of Pelton wheel in following way. The net head $h$ supplied to the turbine is estimated as follows.

$h=h^{\prime \prime}+k \frac{V_{2}^{2}}{2 g}+m \frac{V_{2}^{2}}{2 g}+k^{\prime \prime} \frac{V_{1}^{2}}{2 g}$

where $h$ " is the head converted into mechanical work, the second term represents the energy dissipated from the heat due to internal friction and eddy losses within the runner, the third term is the kinetic energy loss at the discharge, and the fourth term represents the loss in the nozzle of a Pelton wheel.

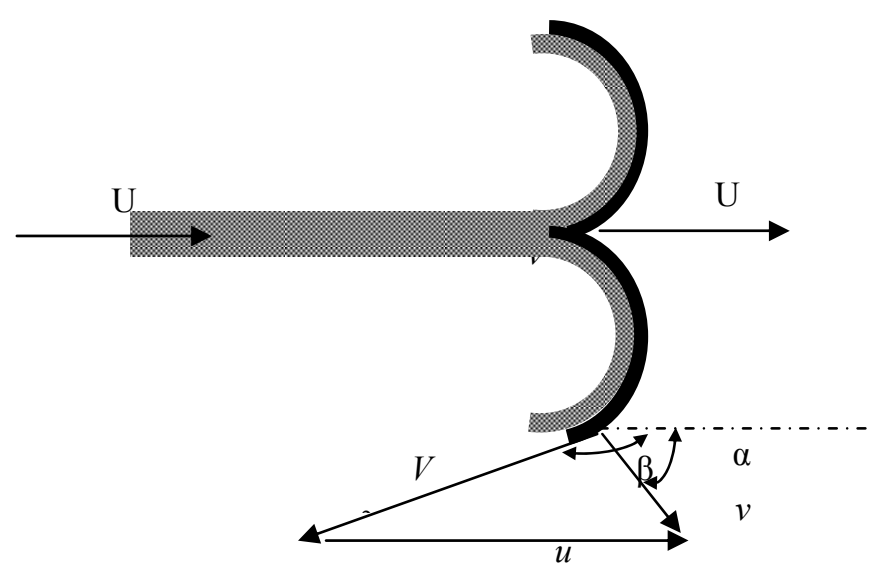

Fig. 2 Schematic diagram of velocity components of flow inside a Pelton Bucket

Head utilized by runner, $h^{\prime \prime}=\frac{1}{g}\left(u_{1} v_{1}-u_{2} v_{2} \cos \alpha_{2}\right)$

Using equation of energy for relative motion of the water in the runner

The total head loss within the runner

$$
\left(Z_{1}+\frac{V_{1}^{2}}{2 g}+\frac{P_{1}}{\rho g}\right)-\left(Z_{2}+\frac{V_{2}^{2}}{2 g}+\frac{P_{2}}{\rho g}\right)
$$

Using relative velocities and eqns (1) and (2)

$\left(Z_{1}+\frac{V_{1}^{2}-U_{1}^{2}}{2 g}+\frac{P_{1}}{\rho g}\right)-\left(Z_{2}+\frac{V_{2}^{2}-U_{2}^{2}}{2 g}+\frac{P_{2}}{\rho g}\right)=k \frac{V_{2}^{2}}{2 g}$

For the Pelton wheel assuming $Z_{1}=Z_{2}, P_{1}=P_{2}, u_{1}=u_{2}$

$V_{2}=\frac{V_{1}}{\sqrt{1+k}}=\frac{v_{1}-U_{1}}{\sqrt{1+k}}$

Tangential force on the bucket $F=\dot{m}\left(U-u_{2}-V_{2} \cos \beta_{2}\right)$

$F=\dot{m}\left(1-\frac{\cos \beta_{2}}{\sqrt{1+k}}\right)\left(V_{1}-U_{1}\right)$

Power developed $P=\dot{m}\left(1-\frac{\cos \beta_{2}}{\sqrt{1+k}}\right)\left(V_{1}-U_{1}\right) U_{1}$

In real situation it cannot be assumed that $\mathrm{U}=v_{l}$ and always jet is striking the bucket at an angle. 


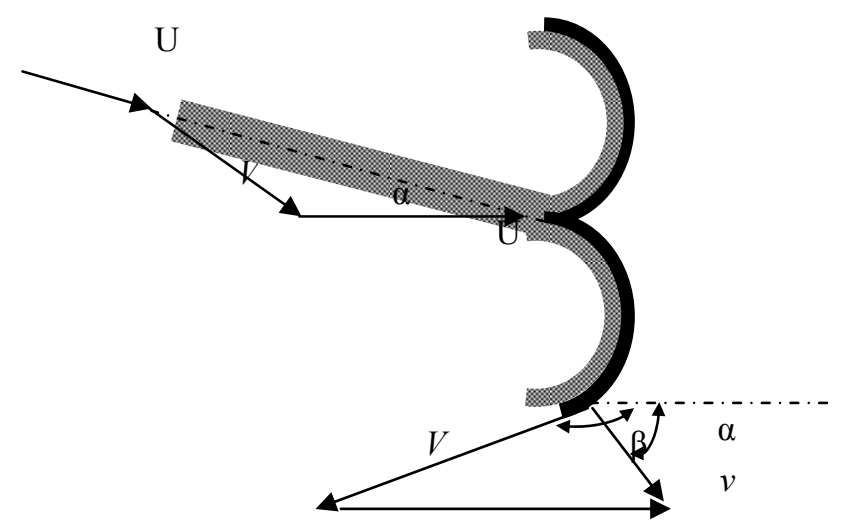

Fig. 3 Considering all the real situations the equation for power developed by the Pelton turbine is

$P=\dot{m}\left[U \cos \alpha_{1}-x^{2} U_{1}-\frac{x \cos \beta_{2}}{\sqrt{1+k}} \sqrt{U^{2}-2 U U_{1} \cos \alpha_{1}+x^{2} U_{1}^{2}}\right]$ where $x=\frac{r_{1}}{r_{2}}$

The value of $k$ was identified as purely empirical value. The value of $\mathrm{k}$ depends mainly on the frictional factor along the bucket surface and eddy losses.

An analysis carried out on design of small water turbines by Mahomadd Duralli has also identified two type of losses that occur in the turbine blade passage. One is hydraulic friction loss in the blade and the other one is loss due to flow direction change. The hydraulic friction loss $\left(\mathrm{h}_{\mathrm{L}}\right)$ is estimated as follows.

$h_{L}=f \frac{L}{D_{h}} \frac{v_{2}}{2 g}$

Where $L$ is the length of the blade $D_{h}$ is the hydraulic diameter

$D_{h}=\frac{4 \times \text { flow area }}{\text { wetted perimeter }}$

And further it is identified that hydraulic loss through the blade is a function of radius of curvature of the blade, hydraulic diameter and deflection angle of the blade. The main difficulty faced is to find the flow area and the wetted perimeter because analysis of water flow in the bucket is extremely complex. Most of the recent studies that have been done were mainly focused on analysis of flow pattern in the turbine bucket both numerically and experimentally. Because it is revealed that the performance of Pelton turbine is mainly depend on the flow variation along the bucket surface. Unlike in the reaction turbines the hydraulic performance is dynamic due to the unsteady flow in the bucket. So many analytical studies have been done over the years. A very recent comprehensive analysis carried out by the Zh Zhang[9] has done a detail analysis of the control forces in the relative flow of water in the bucket. The relative motion of water in the bucket is affected by the centrifugal, Coriolis and friction forces acting on the free surface flow and it is proven that the effect of centrifugal and Coriolis forces can be neglected.

\section{Problem Analysis}

The power reduction due to friction force in the Pelton turbine bucket can be analysed by using the Boundary layer theory. Due to the flow along the bucket surface the boundary layer is formed and retards the velocity of the flow.

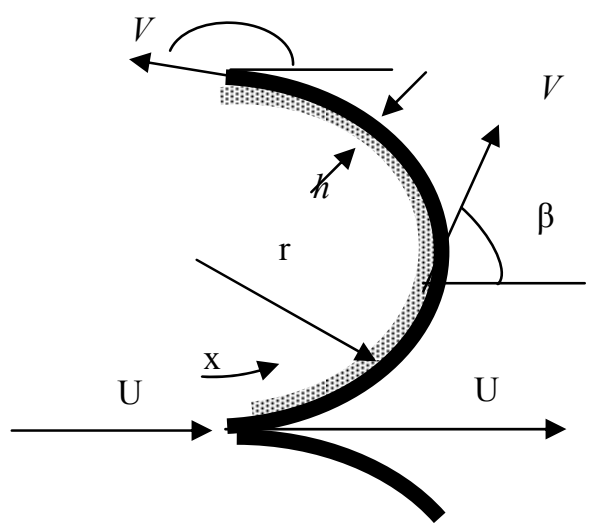

Fig. 4 i Schematic diagram of flow around Pelton Turbine Bucket-Side View 


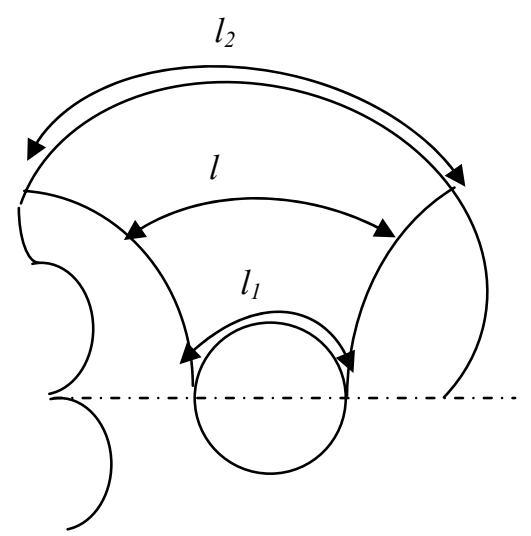

Fig. 4 ii Schematic diagram of flow around Pelton Turbine Bucket-Plan View

Applying the momentum flow equation considering only the frictional forces, to the water flow along the bucket it can be written[0],

$\rho \operatorname{lhV} d v=-c_{f} \frac{1}{2} \rho V^{2} l . d x$

$V d v=-c_{f} \frac{1}{2 h} \rho V^{2} \cdot d x$

The skin friction coefficient is approximately a constant at large Reynolds numbers $(0)$. Assuming $\mathrm{c}_{\mathrm{f}}$ is a constant value, equation (4) can be written as

$\int_{V_{1}}^{V_{2}} V d V=-c_{f} \int_{0}^{X} \frac{1}{2 h} \rho V^{2} \cdot d x$

The volume flow rate in the nozzle $\dot{Q}_{m}$

$\dot{Q}_{m}=\frac{\pi D^{2}}{4} U$

The volume flow rate in the bucket is $\dot{Q}_{w}$

$\dot{Q}_{w}=\frac{\dot{Q}_{m}}{Z}$

Where

$Z=\frac{U}{V}$

$\dot{Q}=V_{1} h_{1} l_{1}$

at $x=0 ; h=h_{1}$

at $x=L ; h=h_{2}$

For one side of the bucket

$\dot{Q}=\frac{\pi D^{2}}{8} V_{1}$

From the continuity equation

$V_{1} h_{1} l_{1}=V_{2} h_{2} l_{2}$

$h_{1}=\frac{\dot{Q}}{V_{1} l_{1}}$

$h_{2}=\frac{\dot{Q}}{V_{2} l_{2}}$

From the numerical and experimental investigations done in literature assuming the thickness of the water sheet vary linearly along the flow path[7] 
$h=h_{1}-\frac{\left(h_{1}-h_{2}\right)}{L} x$

$h=\lambda_{1}-\lambda_{2} x$

Where

$\lambda_{1}=h_{1}$

And

$\lambda_{2}=\frac{\left(h_{1}-h_{2}\right)}{L}$

Substituting $h$ in equation (3)

$\int_{V_{1}}^{V_{2}} V d V=\frac{-c_{f}}{2} \int_{0}^{L} \frac{1}{\lambda_{1}-\lambda_{2} x} \rho V^{2} \cdot d x$

$\int_{V_{1}}^{V_{2}} V d V=\frac{-c_{f}}{2 \lambda_{1}} \int_{0}^{L} \frac{1}{1-\lambda_{3} x} \rho V^{2} \cdot d x$

where

$\lambda_{3}=\frac{\lambda_{2}}{\lambda_{1}}$

$[\ln V]_{V_{1}}^{V}=\frac{-c_{f}}{2 \lambda_{1}} \int_{0}^{L} 1+\lambda_{3} x+\left(\lambda_{3} x\right)^{2}+\left(\lambda_{4} x\right)^{4} d x$

Let

$A=\frac{-c_{f}}{2 \lambda_{1}} \int_{0}^{L} 1+\lambda_{3} x+\left(\lambda_{3} x\right)^{2}+\left(\lambda_{4} x\right)^{4} d x$

Therefore

$V=V_{1} e^{A}$

Assuming water flow in the turbine bucket linearly vary along the flow path the following expression can be written.

$l=l_{1}+\frac{\left(l_{2}-l_{1}\right)}{L} x$

Now the power dissipated due to direct friction can be written as in equation

$\dot{P}_{f}=\int_{0}^{L}-c_{f} \frac{1}{2} \rho V^{2} U_{1} \cos \beta l d x$

$\dot{P}_{f}=\frac{-\rho c_{f} U_{1}}{2} \int_{0}^{L} V^{2} U_{1} \cos \beta l d x$

Substituting values from equation (13) and (14)

$\dot{P}_{f}=\frac{-\rho c_{f} V_{1}^{2} U_{1}^{2}}{2} \int_{0}^{L} e^{2 A} \cos \beta l d x$

Equation (16) can be written as

$\dot{P}_{f}=B \frac{-\rho c_{f} V_{1}^{2} U_{1}^{2}}{2}$

Where

$B=\int_{0}^{L} e^{2 A} \cos \beta l d x$ 
Integration of all the equation are done using EES (Engineering equation solver)

Therefore the total power dissipation due to direct friction can be written as

$\dot{P}_{f}=Z B \frac{-\rho c_{f} V_{1}^{2} U_{1}^{2}}{2}$

(17)

The water flows along the bucket and because of the shape of the bucket the relative velocity is continuously subjected to the direction change. The over pressure on the bucket is estimated as follows as in reference[10].

$p_{b}=\frac{\rho}{r} h V^{2}$

The force due to the pressure distribution acts normal to the bucket surface. The circumferential component of the force is taken for the calculation.

$d P_{p}=p_{b} n U_{1} l d x$

$d P_{p}=\frac{\rho}{r} h V^{2} U_{1} \sin \beta l d x$

$\dot{m}_{w}=\rho V h l$

$d P_{p}=\dot{m}_{c} U_{1} V \frac{1}{r} \sin \beta d x$

Total power dissipation

$P_{p}=\int_{0}^{L} \dot{m}_{w} U_{1} V \frac{1}{r} \sin \beta d x$

$P_{p}=\frac{\dot{m}_{w} U_{1}}{r} \int_{0}^{L} V \sin \beta d x$

$\beta=\frac{x}{r}$

Substituting V from equation (13)

$P_{p}=\frac{\dot{m}_{w} U_{1}}{r} \int_{0}^{L} V_{1} e^{A} \sin \beta d x$

$P_{p}=\frac{\dot{m}_{w} U_{1} V_{1}}{r} \int_{0}^{L} e^{A} \sin \beta d x$

Therefore power dissipated due to pressure variation can be written as

$P_{p}=D \frac{\dot{m}_{w} U_{1} V_{1}}{r}$

Where

$\mathrm{D}=\int_{0}^{L} e^{A} \sin \beta d x$

Frictionless flow (from direct integration)

If the flow is frictionless the magnitude of the relative velocity is not subjected to change.

$V=V_{1}$

From equation (17)

$P_{p}=\frac{\dot{m}_{w} U_{1} V_{1}}{r} \int_{0}^{L} \sin \beta d x$ 
$P_{p}=\frac{\dot{m}_{w} U_{1} V_{1}}{r} \int_{0}^{L} \sin \beta d x$

$P_{p}=\frac{\dot{m}_{w} U_{1} V_{1}}{r}\left(1-\cos \beta_{2}\right)$

Where $\beta_{2}$ is the bucket exit angle.

Frictionless flow (from derived Mathematical model)

If there is no friction $\mathrm{C}_{\mathrm{f}}=0, \mathrm{~A}=0$

Therefore from equation (21)

$e^{A}=1$

$\mathrm{D}=\int_{0}^{L} e^{A} \sin \beta d x$

$P_{p}=\frac{\dot{m}_{w} U_{1} V_{1}}{r} \int_{0}^{L} \sin \beta d x$

The total power dissipated can be written as

$P_{p}=\frac{\dot{m} U_{1} V_{1}}{r}\left(1-\cos \beta_{2}\right)$

Frictionless flow (from derived Mathematical model)

If there is no friction $\mathrm{C}_{\mathrm{f}}=0, \mathrm{~A}=0$

Therefore from equation (21)

$e^{A}=1$

$\mathrm{D}=\int_{0}^{L} e^{A} \sin \beta d x$

$P_{p}=\frac{\dot{m}_{w} U_{1} V_{1}}{r} \int_{0}^{L} \sin \beta d x$

$P_{p}=\frac{\dot{m} U_{1} V_{1}}{r} \int_{0}^{L} \sin \beta d x$

If the flow is frictionless the equation for power dissipated due to pressure change is same as the equation that is obtained by direct integration.

Therefore the power loss due to indirect effect of friction is

$P_{i n}=\left(\frac{\dot{m} U_{1} V_{1}}{r} \int_{0}^{L} \sin \beta d x\right)-\frac{\dot{m} U_{1} V_{1}}{r} \int_{0}^{L} e^{A} \sin \beta d x$

$P_{i n}=\left(\frac{\dot{m} U_{1} V_{1}}{r}\left(1-\cos \beta_{2}\right)\right)-\left(D \frac{\dot{m} U_{1} V_{1}}{r}\right)$

\section{Methodology}

Surface roughness of the buckets was varied by adhering sands of different grading, on the bucket. First the Pelton turbine was tested with its original conditions of the surfaces of the buckets. Then sand was adhered to the bucket using super glue. After carrying out test for one grading of sand, Pelton runner was kept in acetone bath to remove the sand about 2 days. The above procedure was repeated for different spear openings of the water issuing nozzle. Sand of particle sizes $150 \mu \mathrm{m}, 212 \mu \mathrm{m}, 425 \mu \mathrm{m}$ and $600 \mu \mathrm{m}$ were pasted on buckets and experimental values of power loss was obtained. 


\section{Test Rig}

All the measurements are carried out on the horizontal Pelton test rig available in the civil Engineering laboratory at the Open University of Sri Lanka. Fig. 5 i shows detail technical drawing of the Pelton runner that was tested and Fig 5 ii shows the detail technical drawing of the nozzle.

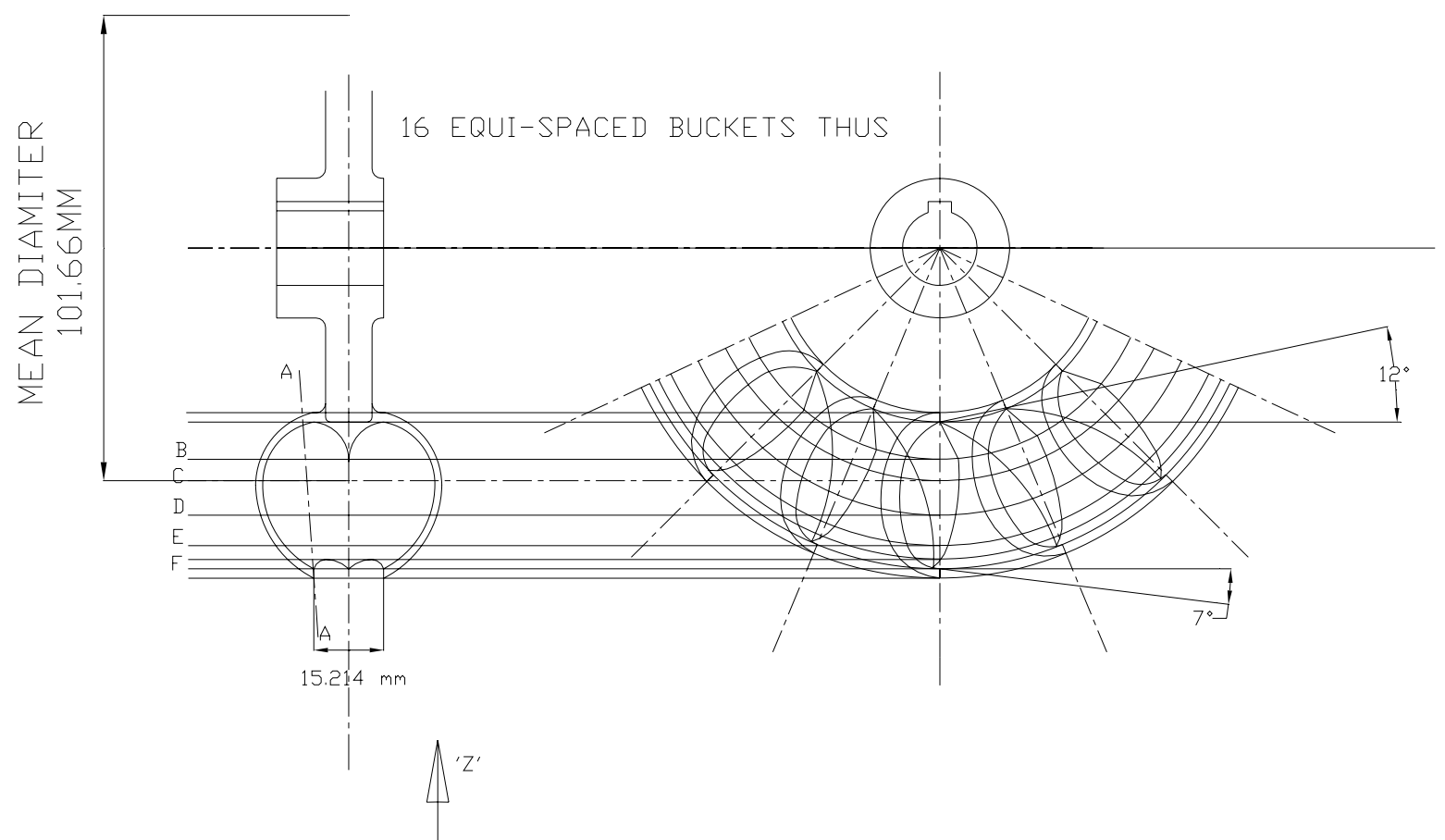

Fig. 5 i Technical details of the Pelton runner

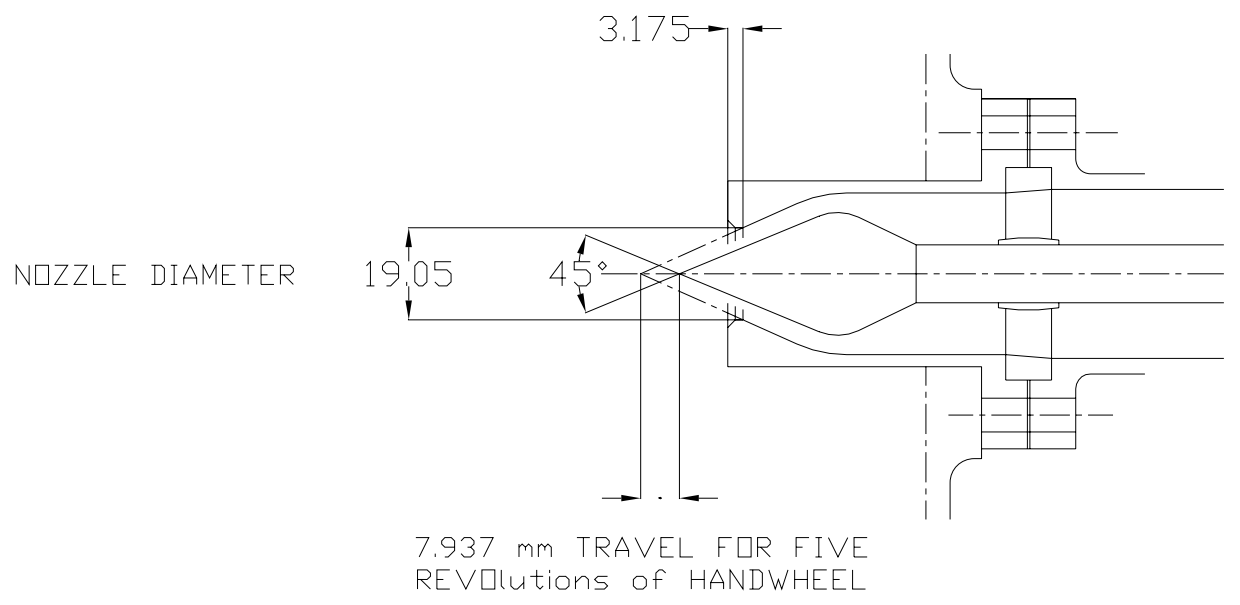

Fig 5 ii Section through nozzle

Pressure is measured by means of the Bourdon gauge. And speed is measured by tachometer. The pony brake is used to apply a load on the rotating shaft of the turbine and force gauge is used to measure the force. The discharge is measured using orifice meter. The surface roughness tester was used to verify the relative roughness of the surface of the Pelton turbine buckets after pasting sand. 


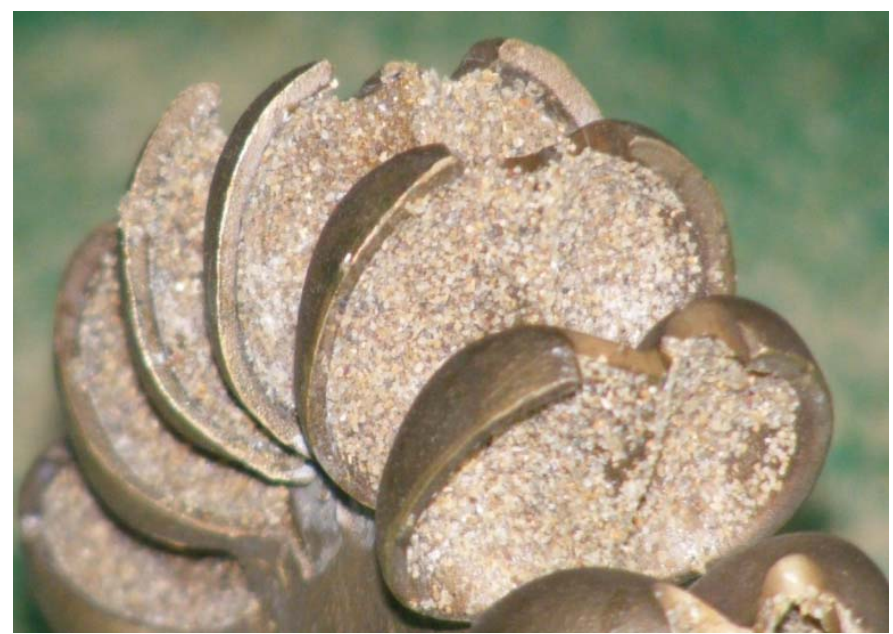

Fig. 6 Closer view of Pelton runner after pasting sand

\section{Analysis and Results}

At the beginning original runner was tested and at the brake loads of $3 \mathrm{~N}, 6 \mathrm{~N}, 9 \mathrm{~N}$, and $12 \mathrm{~N}$ the experimental power developed by the Pelton turbine was recorded. After that by varying the surface roughness of the buckets, power developed by the turbine in each trial for the same brake loads were also measured and hence the percentage power loss was calculated. The theoretical values of percentage power loss in each trial were calculated by using equations (17) and (26). To solve these equations EES (Engineering equation solver) software was used and programme window is shown in Figure 7. From Figure 8 and Figure 9 it can be seen that when the surface roughness increases, the percentage power loss due to direct effect of friction and indirect effect of friction are also increase for all applied loads on the turbine. At the same time the percentage power loss decreases when the turbine is loaded since the speed of the turbine decreases when loading the turbine. Figure 10 shows variation of total predicted percentage power loss.

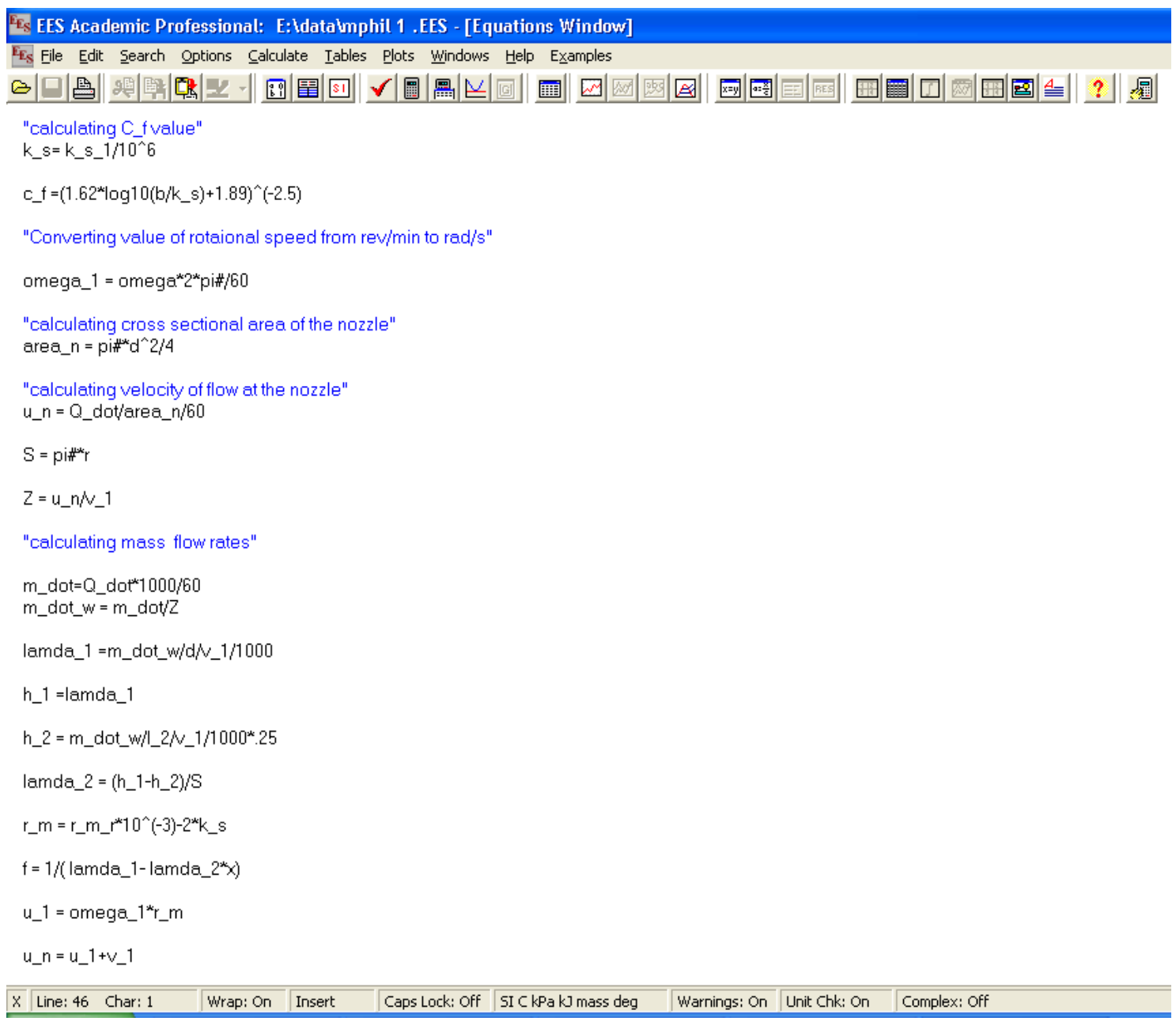

Fig 7 Programme for solving mathematical model in EES software 


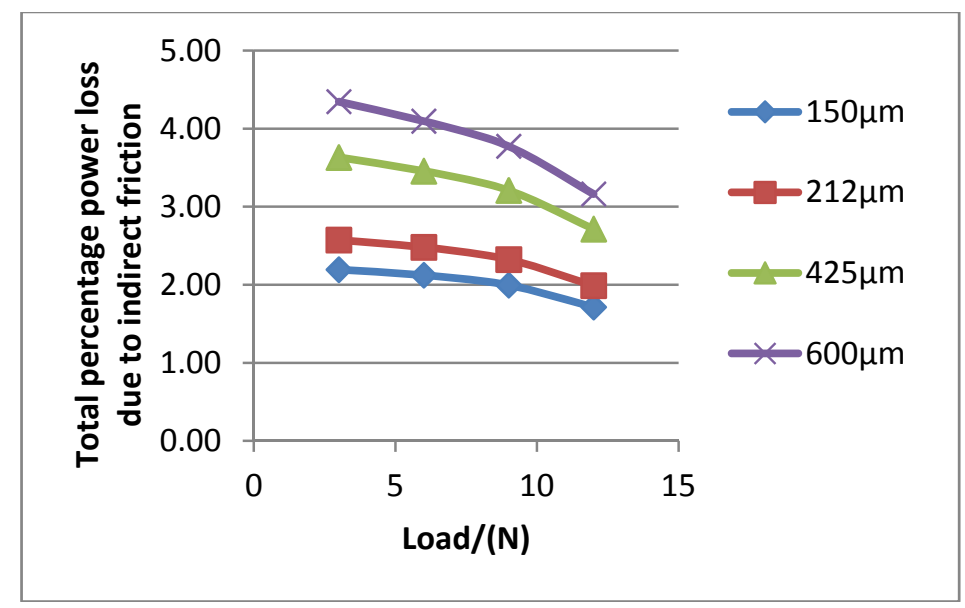

Fig 8 Variation of percentage power loss with load for different roughness values due to indirect effect of friction

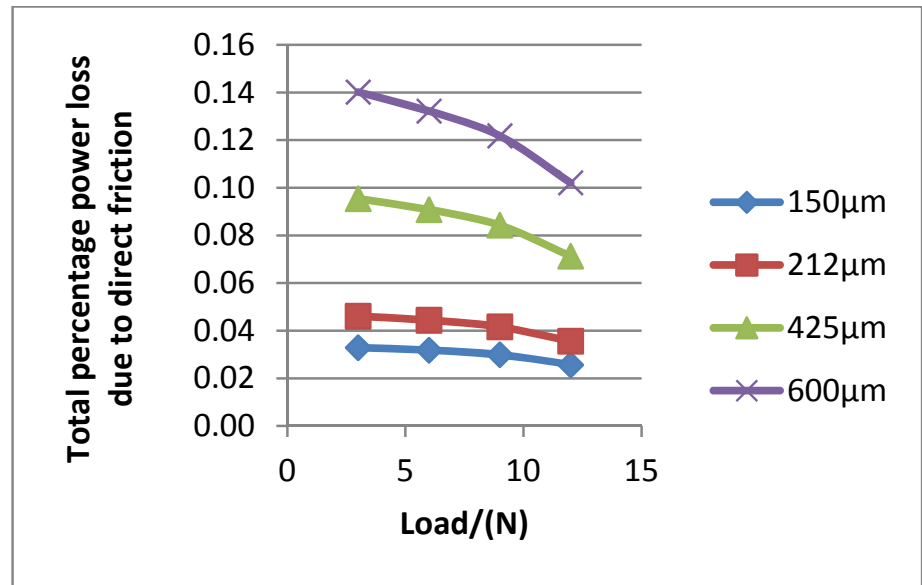

Fig 9 Variation of percentage power loss with load for different roughness values due to direct effect of friction

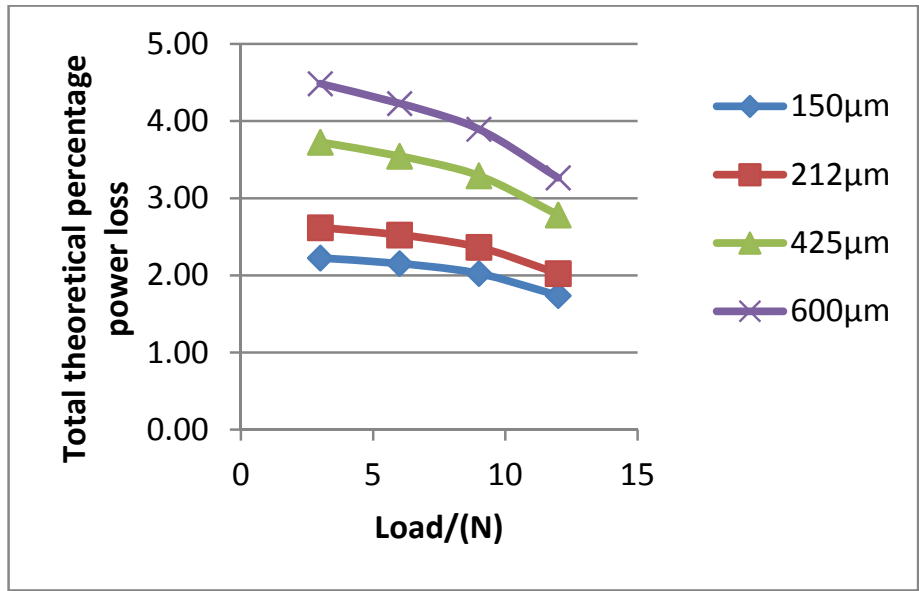

Fig. 10 Variation of total theoretical percentage power loss with load for different roughness values

Figure 11 shows variation of predicted percentage power loss due to direct and indirect friction in all trials of the experiment. The surface roughness values are indicated in the graph. Figure 12 shows experimental value of percentage power loss and the theoretically predicted values of percentage power loss for all trials. 


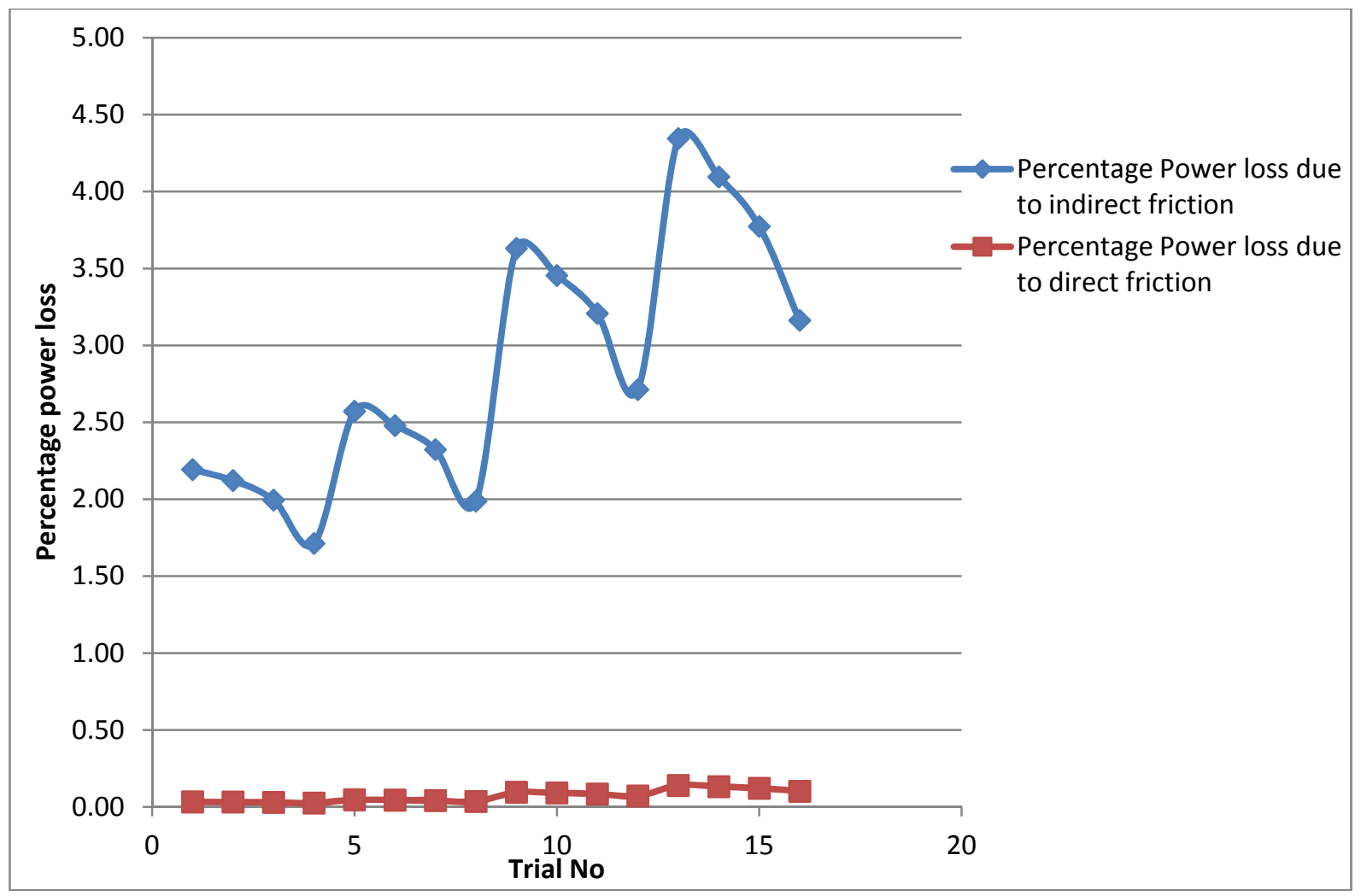

Fig. 11 Variation of percentage power loss due to direct friction and indirect effect of friction

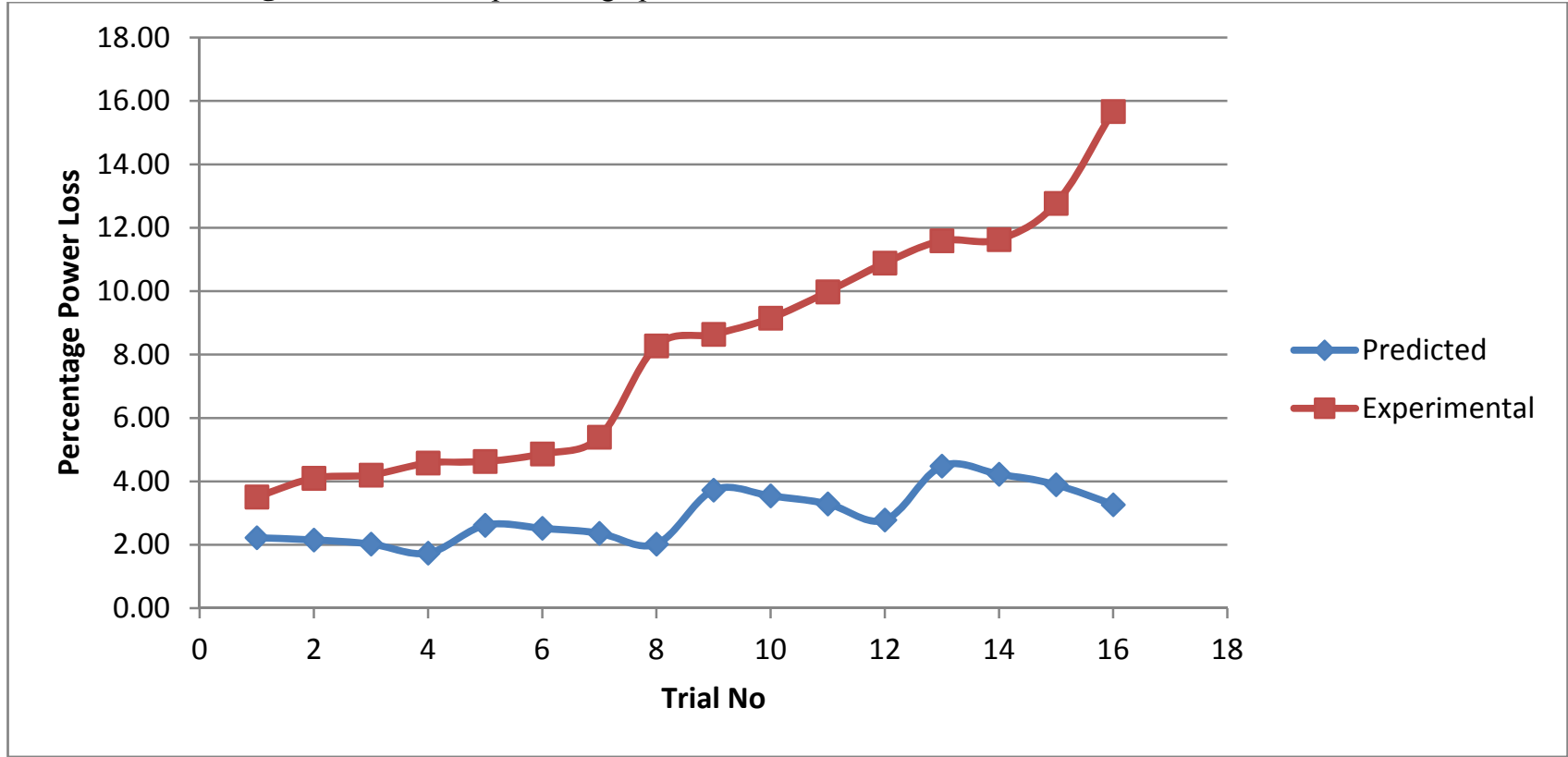

Fig 12 Variation of Experimental and theoretically predicted percentage power loss

The main difference that can be stated from the comparison of the experimental and the theoretically predicted values is as follows: theoretically predicted values for percentage power loss for all spear openings increases when roughness of the bucket increases and decreases with speed of the runner decreases or load on the runner increases. But for experimental values, the percentage power loss increases when roughness height increases and also it increases when speed of the runner decreases.

When theoretically predicting values for power loss, only the surface roughness effect is considered whereas in the real experiment power is also lost due to some other effects.

When sand of different sizes was pasted along the bucket surface the thickness of the splitter of the bucket increases creates another power loss in the system. In order to reduce losses occur at the splitter, the splitter edge has to be a smooth one with almost no thickness. The percentage power loss due to this effect can be estimated as follows. 


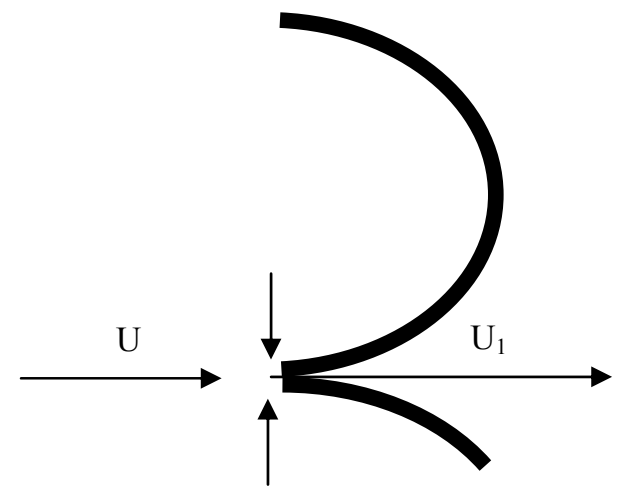

Fig 13

From the force momentum equation power loss by hitting the jet portion on splitter can be written as

$P_{s p}=\frac{1}{2} \dot{m}_{s p}\left(U-U_{1}\right)^{2}$

$\dot{m}_{s p}=t D U$

Equation (25) indicates that the loss of power at the splitter increases when speed of the Pelton runner or the turbine increases. Figure 13 shows variation of total predicted power loss including loss of power at the splitter. When this particular effect is considered the experimental values and theoretical values of percentage power loss varying in the same manner.

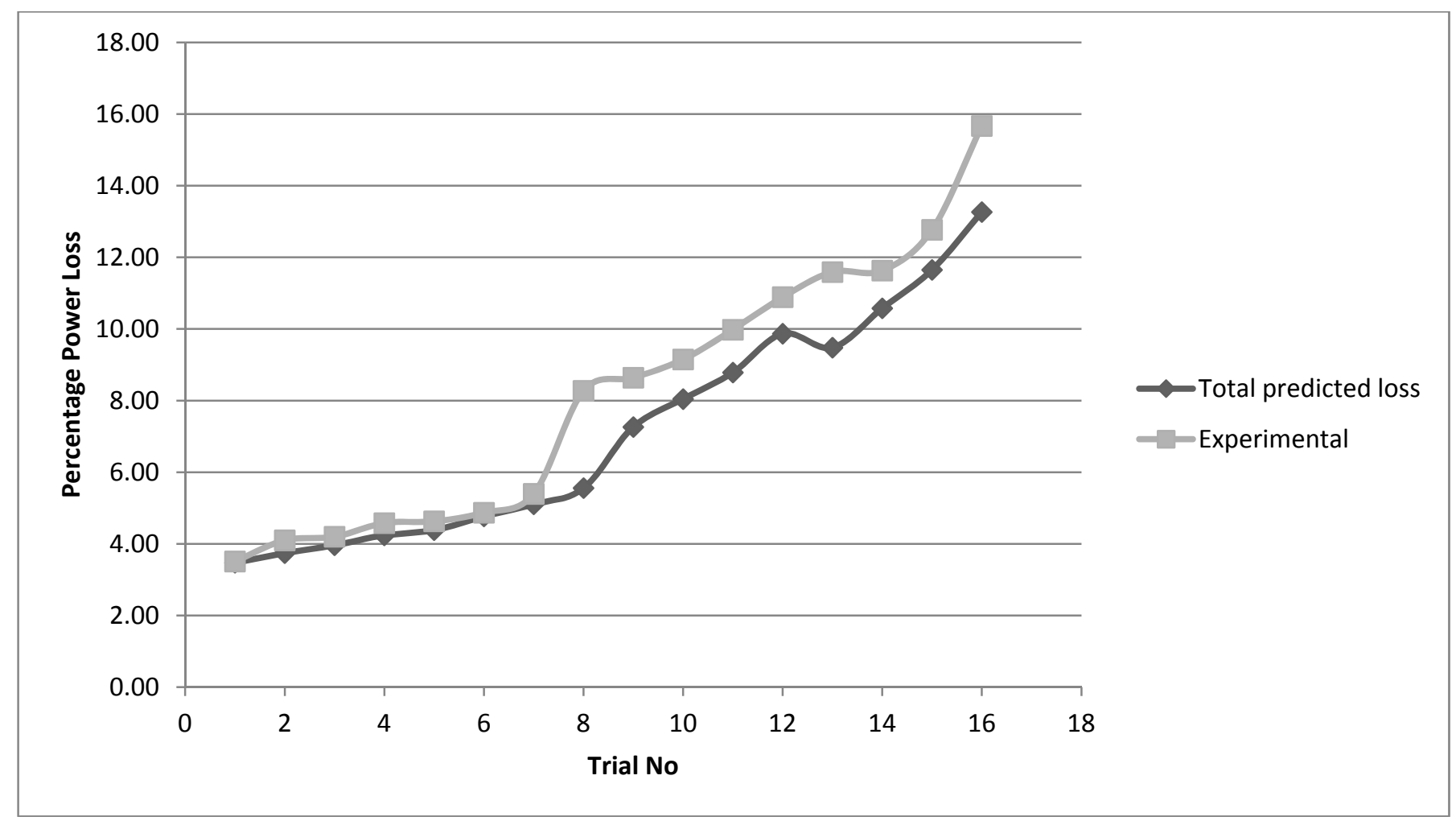

Fig 14 Variation of experimental and total predicted percentage power loss.

\section{Conclusion}

When the water flow inside the Pelton turbine is considered the power loss can be occurred due to friction in the bucket surface. The power loss due to direct effect of friction that is due to change in the velocity of the water steam when flowing along the bucket surface as modeled in equation (17). The indirect effect of friction is modeled in equation (26). The indirect effect of friction on performance of Pelton turbine is much more higher than that of the direct friction. The total percentage power loss increases when rotational speed of the turbine increases. Due to the method of increasing surface roughness of the bucket for this particular set of experimental data an additional power loss occurs at the splitter of the bucket. When the power loss at the splitter 
is included to the predicting values of percentage power loss experimental values and theoretical values are closer to each other which validate the two mathematical models. The major assumption that made in the analysis is flow thickness along the Pelton turbine bucket varies linearly which is partially validated in the previous studies [5]. Another assumption made is the width of the flow sheet also linearly very along the flow path which is also addressed in few publications [4], [7].

\section{Acknowledgments}

This project is funded by Distance education modernization project (DEMP) another project of Asian development Bank. Special thanks for the technical support given by Mr. K.C.K Deraniyagala a Technical officer in the Department of Mechanical Engineering, The Open University of Sri Lanka.

\section{Nomenclature}

\begin{tabular}{|c|c|c|c|}
\hline$c_{f}$ & Coefficient of friction & $\mathrm{R}$ & Mean radius of the runner \\
\hline $\mathrm{D}$ & Diameter of the jet & $\mathrm{t}$ & Thickness of the splitter \\
\hline$D_{h}$ & Hydraulic diameter & $\mathrm{U}$ & Velocity of the jet \\
\hline $\mathrm{F}_{\mathrm{co}}$ & Coriolis force & $\mathrm{U}_{1}$ & Velocity of the Pelton runner \\
\hline $\mathrm{F}_{\mathrm{ct}}$ & Centrifugal force & $V$ & Relative velocity of water \\
\hline $\mathrm{h}$ & Thickness of the flow sheet & $v_{1}$ & Absolute velocity of water at the entrance to the bucket \\
\hline $\mathrm{H}$ & Water head & $V_{1}$ & Relative velocity of water at the entrance to the bucket \\
\hline$l$ & Width of the flow sheet & $v_{2}$ & Absolute velocity of water at the exit from the bucket \\
\hline $\mathrm{L}$ & Total distance of the flow path & $V_{2}$ & Relative velocity of water at the exit from the bucket \\
\hline$l_{1}$ & Width of the flow sheet at the inlet & $v_{\mathrm{f}, \mathrm{o}}$ & velocity of flow at outlet \\
\hline$l_{2}$ & Width of the flow sheet at the outlet & $v_{\mathrm{w}, \mathrm{o}}$ & velocity of whirl at outlet \\
\hline $\mathrm{P}_{\mathrm{b}}$ & Over pressure on the bucket & $v_{\mathrm{f}, \mathrm{I}}$ & velocity of flow at inlet \\
\hline $\mathrm{P}_{\mathrm{co}}$ & Power dissipated due to coriolis force & $v_{\mathrm{w}, \mathrm{o}}$ & velocity of whirl at inlet \\
\hline $\mathrm{P}_{\mathrm{ct}}$ & Power dissipated due to Centrifugal force & $\mathrm{x}$ & Distance measured along the flow path \\
\hline $\mathrm{P}_{\mathrm{f}}$ & Power dissipated due to direct friction & $\beta$ & Blade angle \\
\hline$P_{\text {in }}$ & $\begin{array}{l}\text { Power dissipated due to indirect effect of } \\
\text { friction }\end{array}$ & $\dot{m}$ & Mass flow rate from the nozzle \\
\hline$P_{s p}$ & Power loss in the splitter & $\dot{m}_{w}$ & Mass flow rate in the bucket \\
\hline $\mathrm{r}$ & Radius of the bucket & $\dot{m}_{s p}$ & Mass flow rate that hitting the splitter \\
\hline
\end{tabular}

\section{References}

[1] Adriana Catanase, Mircea BĂRGLĂZAN, Cristina HORA, 2004, "Numerical Simulation Of A Free Jet In Pelton Turbine," The 6th International Conference on Hydraulic Machinery and Hydrodynamics Timisoara, Romania, October 21 - $22,2004$.

[2] Avellan, F., Dupont, P., Kvicinsky, S., Chapuis, L., Parkinson, E., and Vullioud, G., "Flow calculations in Pelton turbines - part 2: Free surface flows", Proceedings of the 19th IAHR Symposium.

[3] Draughty M., 1920, "Hydraulic Turbines," Mc Graw Hill Book Company, Third Edition.

[4] Grozev, G., Obretenov, V., and Trifonov, T., "Investigation of the distribution of pressure over the buckets of a pelton turbine," In Velensek and Bajd [110]. Proceedings of the conference on hydraulic machinery and flow measurements.

[5] Hana, M., "Improvement of a graphical method for calculation of flow on a pelton bucket," In Hydraulic Machinery and Cavitation (Dordrecht, NL, 1996), E. Cabrera, V. Espert, and F. Martinez, Eds., vol. 1, Kluwer Academic Publishers. Proceedings of the XV III IAHR Symposium.

[6] Kvicinsky, S., Kueny, J., Avellan, F., and Parkinson, E., 2002, "Experimental and numerical analysis of free surface flows in a rotating bucket," In 21st IAHR Symposium on hydraulic Machinery and Systems, Lausanne, Switzerland.

[7] Parkinson, E., Vuilloud, G., Geppert, L., and Keck, H., 2002, “Analysis of pelton turbine flow patterns for improved runnercomponent interaction," Hydropower \& Dams, Issue Five.

[8] Perrig, A., Farhat, M., Avellan, F., Parkinson, E., Garcin, H., Bissel, C., Valle, M., and Favre, J., "Numerical flow analysis in a pelton turbine bucket," In Hydraulic Machinery and Systems [49]. Proceedings of the 22nd IAHR Symposium.

[9] Zhang, Zh., 2007, "Flow interactions in Pelton turbines and the hydraulic efficiency of the turbine system," J. Power Energy.

[10] Zh Zhang, 2009, "Flow dynamics of the free surface flow in the rotating buckets of a Pelton turbine," Proc. IMechE Vol. 223

Part A: J. Power and Energy. 